\title{
THE WEDDERBURN PRINCIPAL THEOREM FOR ALTERNATIVE ALGEBRAS
}

\author{
R. D. SCHAFER
}

Except for a generalization of the so-called Wedderburn principal theorem, the structure theory of alternative algebras over an arbitrary field is as complete as that for associative algebras. It is our purpose here to fill this one gap in the alternative theory.

1. The principal theorem. A non-associative algebra $\mathfrak{A}$ of order $n$ over an arbitrary field $\mathfrak{F}$ is called alternative in case

$$
a x^{2}=(a x) x, \quad x^{2} a=x(x a)
$$

for all $a, x$ in $\mathfrak{A}$. It is clear that associative algebras are alternative.

The most famous examples of alternative algebras which are not associative are the so-called Cayley-Dickson algebras of order 8 over $\mathfrak{F}$. Let $\$$ be an algebra of order 2 over $\mathfrak{F}$ which is either a separable quadratic field over $\mathfrak{F}$ or the direct sum $\mathfrak{F} \oplus \mathfrak{F}$. There is one automorphism $z \rightarrow \bar{z}$ of $\mathscr{Z}$ (over $\mathfrak{F}$ ) which is not the identity automorphism. The associative algebra $\mathfrak{Q}=\mathfrak{B}+u \mathfrak{B}$ with elements

$$
q=z_{1}+u z_{2}, \quad z_{i} \text { in } 3,
$$

and multiplication defined by

$$
\left(z_{1}+u z_{2}\right)\left(z_{3}+u z_{4}\right)=\left(z_{1} z_{3}+\beta z_{4} \bar{z}_{2}\right)+u\left(\bar{z}_{1} z_{4}+z_{3} z_{2}\right)
$$

for $\beta \neq 0$ in $\mathfrak{F}$ is called a quaternion algebra. For $q$ in the form (1), the correspondence

$$
q \rightarrow \bar{q}=\bar{z}_{1}-u z_{2}=t(q)-q
$$

is an involution of $\mathfrak{Q}$. The Cayley-Dickson algebras $\mathfrak{S}=\mathfrak{Q}+v \mathfrak{Q}$ are obtained by repetition of this process: the elements of $\mathbb{S}$ are

$$
x=q_{1}+v q_{2}
$$

$q_{i}$ in $\mathfrak{D}$,

and multiplication in $\mathbb{5}$ is defined by

$$
\left(q_{1}+v q_{2}\right)\left(q_{3}+v q_{4}\right)=\left(q_{1} q_{3}+\gamma q_{4} \bar{q}_{2}\right)+v\left(\bar{q}_{1} q_{4}+q_{3} q_{2}\right)
$$

for $\gamma \neq 0$ in $\mathfrak{F}$, where $q \rightarrow \bar{q}$ is the involution (3) of $\mathfrak{Q}$.

Most of our knowledge of alternative algebras is due to M. Zorn. ${ }^{1}$

Presented to the Society, April 17, 1948; received by the editors February 9, 1948, and, in revised form, April 26, 1948.

${ }^{1}$ See references [6], [7], [9]. Numbers in brackets refer to the references cited at the end of the paper. 
The radical $\mathfrak{N}$ of an alternative algebra $\mathfrak{A}$ is the set of properly nilpotent elements of $\mathfrak{A}$, and is the maximal nilpotent ideal ${ }^{2}$ of $\mathfrak{A}$. The difference algebra $\mathfrak{A}-\mathfrak{N}$ is semi-simple: it is the direct sum $\mathfrak{S}_{1} \oplus \ldots$ $\oplus \mathfrak{S}_{m}$ of simple components $\mathfrak{S}_{i}$. Any simple alternative algebra is central simple (that is, simple for all scalar extensions) over its center, and all central simple alternative algebras are either associative or Cayley-Dickson algebras. ${ }^{3}$ In the sense of equivalence there is (over a given field) exactly one Cayley-Dickson algebra with divisors of zero. $^{4}$

A non-associative algebra $\mathfrak{A}$ over $\mathfrak{F}$ is called separable in case $\mathfrak{A}_{\mathfrak{R}}$ is semi-simple (that is, a direct sum of simple algebras) for every scalar extension $\Re$ of $\mathfrak{F}$. It is easy to see that an alternative algebra $\mathfrak{A}$ is separable if and only if it is the direct sum of simple components whose centers are separable fields (over $\mathfrak{F}$ ). Furthermore, if $\mathfrak{A}$ is separable, there exists a scalar extension $\Re$ of finite degree over $\mathfrak{F}$ such that $\mathfrak{A}_{\Omega}$ is a direct sum of components each of which is either a total matric algebra or a Cayley-Dickson algebra with divisors of zero. $^{5}$ Such a scalar extension $\mathfrak{\Omega}$ of $\mathfrak{F}$ we call a splitting field of $\mathfrak{A}$, and we use the term split algebra for a total matric algebra or a Cayley-Dickson algebra with divisors of zero. The number of total matric components of $\mathfrak{A}_{\Omega}$ is the same for all splitting fields $\Re$ of $\mathfrak{A}$, and is the sum of the degrees over $\mathfrak{F}$ of the centers of the associative simple components of $\mathfrak{A}$. Similarly the number of Cayley-Dickson components of $\mathfrak{A}_{\Re}$ is independent of the particular splitting field $\mathscr{\Omega}$, and is the sum of the degrees over $\mathfrak{F}$ of the centers of the simple components of $\mathfrak{A}$ which are not associative.

We shall prove the following.

${ }^{2}$ It is asserted in the conclusion of [9] that $\mathfrak{R}$ is nilpotent, and that this may be shown by methods similar to those employed in [8]. Since no proof has subsequently appeared in the literature, and since we require the result, we give a proof in Lemma 1 below.

${ }^{3}$ We have given in $[5, \S \S 1,2]$ a complete account of the structure of simple alternative algebras over an arbitrary field. We assume some familiarity on the part of the reader with this account and with the proof of the Wedderburn principal theorem for associative algebras as given, say, in $[1, \S 3.8]$.

${ }^{4}$ This corresponds to the fact that there is exactly one quaternion algebra over $\mathfrak{F}$ with divisors of zero: namely, the total matric algebra $\mathfrak{M}_{2}$ of degree two. For $\mathfrak{Q}$ is a division algebra (that is, has no divisors of zero) if and only if $Z$ is a field and $\beta$ in (2) is not the norm $z \bar{z}$ of an element $z$ in $\mathbb{Z}$; otherwise $\mathfrak{Q}$ is the total matric algebra $\mathfrak{M}_{2}$. If $\mathfrak{Q}$ is a division algebra, and if $\gamma$ in (5) is not the norm $q \bar{q}$ of an element $q$ in $\mathfrak{Q}$, then $\mathbb{E}$ is a division algebra; otherwise $\mathbb{E}$ is the unique Cayley-Dickson algebra over $\mathfrak{F}$ with divisors of zero.

${ }^{5}$ Since any semi-simple alternative algebra has a unity element, these statements are immediate consequences of $[3, \S 4]$ and $[1, \S 3.7]$. 
THEOREM. Let $\mathfrak{A}$ be an alternative algebra over an arbitrary field $\mathfrak{F}$, and let $\mathfrak{N}$ be the radical of $\mathfrak{A}$. If $\mathfrak{A}-\mathfrak{N}$ is separable, then $\mathfrak{A}=\mathfrak{S}+\mathfrak{N}$ where $\mathfrak{S}$ is equivalent to $\mathfrak{A}-\mathfrak{N}$.

This theorem generalizes to the case of alternative algebras the well-known Wedderburn principal theorem for associative algebras [1, p. 47]. Its proof resembles the associative one.

2. The case $\mathfrak{R}^{2} \neq 0$. We use the fact" that the "associator"

$$
\left[a_{1}, a_{2}, a_{3}\right]=\left(a_{1} a_{2}\right) a_{3}-a_{1}\left(a_{2} a_{3}\right)
$$

"alternates" in an alternative algebra $\mathfrak{A}$; that is,

$$
\left[a_{1}, a_{2}, a_{3}\right]=\epsilon\left[a_{i_{1}}, a_{i_{2}}, a_{i_{3}}\right]
$$

$a_{i}$ in $\mathfrak{A}$,

for any permutation $i_{1}, i_{2}, i_{3}$ of $1,2,3$, where $\epsilon$ is 1 in case the permutation is even, -1 in case it is odd. Furthermore, any subalgebra of $\mathfrak{A}$ generated by only two elements is associative. ${ }^{7}$ This implies that powers $x^{j}$ of an element $x$ in $\mathfrak{A}$ are unambiguously defined, and that we have

$$
R_{x^{j}}=R_{x}^{j}, \quad L_{x^{j}}=L_{x}^{j}, \quad L_{x} R_{x}=R_{x} L_{x}
$$

of all $x$ in $\mathfrak{A}$, where $R_{x}$ and $L_{x}$ are the right and left multiplications ${ }^{8}$ of $\mathfrak{A}$ determined by $x$. Equation (7) is equivalent to the equalities

$$
\begin{aligned}
R_{x} R_{y}-R_{x y} & =L_{x y}-L_{y} L_{x}=L_{y} R_{x}-R_{x} L_{y}=L_{x} L_{y}-L_{y x} \\
& =R_{y} L_{x}-L_{x} R_{y}=R_{y x}-R_{y} R_{x}
\end{aligned}
$$

for all $x, y$ in $\mathfrak{A}$.

A non-associative algebra $\mathfrak{R}$ is called $n$ ilpotent ${ }^{9}$ in case there exists an integer $t$ such that every product of $t$ elements in $\mathfrak{N}$, no matter how associated, is zero. It follows from an observation of Etherington [4, p. 2] that $\mathfrak{N}$ is nilpotent if and only if the associative algebra $\mathfrak{N}^{*}$ generated by the right and left multiplications of $\mathfrak{N}$ is nilpotent (for a simple induction suffices to prove that, if $\mathfrak{N}^{* k}=0$, then every product of $t=2^{k}$ elements of $\mathfrak{N}$ is zero). It is an immediate consequence of the known structure theory for alternative algebras and of the following lemma that the radical $\mathfrak{N}$ of an alternative algebra $\mathfrak{A}$ is the

\footnotetext{
6 [6, equation (5), p. 126].

7 The so-called Theorem of Artin [6, p. 127].

${ }^{8}$ See $[2, \S 2]$.

9 The term "strongly nilpotent" has been used for this concept recently, and "nilpotent" used in case the associative algebra $\mathfrak{N} *$ were nilpotent. However, Etherington's theorem shows the equivalence of the two notions.
} 
maximal nilpotent ideal in $\mathfrak{A}$, and is the minimal ideal such that $\mathfrak{U}-\mathfrak{N}$ is semi-simple.

LEMMA 1 (ZORN). Let $\mathfrak{N}$ be an alternative algebra, each of whose elements is nilpotent. Then $\mathfrak{N}$ is nilpotent.

If $\mathfrak{C}$ is any linear subset of $\mathfrak{N}$, we denote by $\mathfrak{C}^{*}$ the subalgebra of $\mathfrak{N}^{*}$ generated by the right and left multiplications of $\mathfrak{N}$ corresponding to elements of $\mathfrak{c}$. We shall prove by an induction on the number of generating elements of $\mathfrak{B}$ that $\mathfrak{B}^{*}$ is nilpotent for all subalgebras $\mathfrak{B}$; hence, in particular, for $\mathfrak{B}=\mathfrak{R}$. If $\mathfrak{B}$ is generated by one element $x$, then by (8) any $T$ in $\mathfrak{B}^{*}$ is a linear combination of transformations of the form

$$
R_{x}^{j_{1}}, \quad L_{x}^{j_{2}}, \quad R_{x}^{j_{3}} L_{x}^{j_{4}}
$$

for $j_{i} \geqq 1$. Then, if $x^{j}=0$, we have $T^{2 j-1}=0, \mathfrak{B}^{*}$ is nilpotent. Hence, by the assumption of the induction, we may take a maximal proper subalgebra $\mathfrak{B}$ of $\mathfrak{N}$ and know that $\mathfrak{B}^{*}$ is nilpotent. But then there exists an element $x$ not in $\mathfrak{B}$ such that

$$
x \mathfrak{B}^{*} \leqq \mathfrak{B} .
$$

For $\mathfrak{B}^{* k}=0$ implies that $\mathfrak{N B}^{* k}=0 \leqq \mathfrak{B}$, and there exists a smallest integer $m \geqq 1$ such that $\mathfrak{N B} \mathfrak{B}^{* m} \leqq \mathfrak{B}$. If $m=1$, take $x$ in $\mathfrak{N}$ but not in $\mathfrak{B}$; if $m>1$, take $x$ in $\mathfrak{N}^{*} \mathfrak{B}^{-1}$ but not in $\mathfrak{B}$. Then (11) is satisfied. Since $\mathfrak{B}$ is maximal, the subalgebra generated by $\mathfrak{B}$ and $x$ is $\mathfrak{N}$ itself. It follows from (11) that $\mathfrak{N}=\mathfrak{B}+\mathfrak{F}[x]$. Put $y=b$ in (9) for any $b$ in $\mathfrak{B}$. Then (11) implies that

$$
\begin{array}{ll}
R_{x} R_{b}=R_{b_{1}}-R_{b} R_{x}, & R_{x} L_{b}=L_{b} R_{x}+R_{b} R_{x}-R_{b_{2}}, \\
L_{x} R_{b}=R_{b} L_{x}+L_{b} L_{x}-L_{b_{3}}, & L_{x} L_{b}=L_{b_{1}}-L_{b} L_{x}
\end{array}
$$

for $b_{i}$ in $\mathfrak{B}$. Equations (12) show that, in each product of right and left multiplications in $\mathfrak{B}^{*}$ and $(x \mathfrak{F})^{*}$, the transformation $R_{x}$ or $L_{x}$ may be systematically passed from the left to the right of $R_{b}$ or $L_{b}$ in a fashion which, although it may change signs and introduce new terms, preserves the number of factors from $\mathfrak{B}^{*}$ and does not increase the number of factors from $(x \mathfrak{F})^{*}$. Hence any $T$ in $\mathfrak{R}^{*}=(\mathfrak{B}+x \mathfrak{F})^{*}$ may be written as a linear combination of terms of the form (10) and others of the form

$$
B_{1}, \quad B_{2} R_{x}^{m_{1}}, \quad B_{3} L_{x}^{m_{2}}, \quad B_{4} R_{x}^{m_{3}} L_{x}^{m_{4}}
$$

for $B_{i}$ in $\mathfrak{B}^{*}, m_{i} \geqq 1$. Then, if $\mathfrak{B}^{* k}=0$ and $x^{j}=0$, we have $T^{k(2 j-1)}=0$; for every term in the expansion of $T^{k(2 j-1)}$ contains either at least 
$k$ factors $B_{i}$ or an uninterrupted sequence of at least $2 j-1$ factors from $(x \mathfrak{F})^{*}$. Hence $\mathfrak{N}^{*}$ is nilpotent, and so is $\mathfrak{R}$.

Let $\mathfrak{B}$ be an ideal of a non-associative algebra $\mathfrak{A}$. We define a family of subalgebras $\mathfrak{B}^{i}(i=1,2, \cdots)$ of $\mathfrak{A}$ inductively as follows:

$$
\mathfrak{B}^{1}=\mathfrak{B}, \quad \mathfrak{B}^{i}=\left(\mathfrak{B P}^{i-1}, \mathfrak{B}^{i-1} \mathfrak{B}\right), \quad i=2,3, \cdots,
$$

the linear subset of $\mathfrak{A}$ spanned by elements of $\mathfrak{B}_{\mathfrak{B}^{i-1}}$ and $\mathfrak{B}^{i-1} \mathfrak{B}$. For any $\mathfrak{A}$, the $\mathfrak{B}^{i}$ form a descending chain

$$
\mathfrak{B}=\mathfrak{B}^{1} \geqq \mathfrak{B}^{2} \geqq \cdots \geqq \mathfrak{B}^{r} \geqq \cdots
$$

of ideals of $\mathfrak{B}$. For alternative algebras $\mathfrak{A}$, we have the following stronger statement.

Lemma 2. Let $\mathfrak{B}$ be an ideal of the alternative algebra $\mathfrak{A}$. Then the subalgebras $\mathfrak{B}^{i}$ in (13) are ideals of $\mathfrak{A}$.

The proof is by induction: $\mathfrak{B}^{1}$ is an ideal by hypothesis. We assume that $\mathfrak{B}^{i-1}$ is an ideal of $\mathfrak{A}$. An arbitrary element of $\mathfrak{A}_{\mathfrak{B}}{ }^{i}$ is the sum of elements of the form of $a(x y)$ and $a(y x)$ for $a$ in $\mathfrak{A}, x$ in $\mathfrak{B}, y$ in $\mathfrak{B}^{i-1}$. Then

$$
\begin{aligned}
a(x y) & =(a x) y-[a, x, y]=(a x) y-[y, a, x] \\
& =(a x) y-(y a) x+y(a x)
\end{aligned}
$$

by (7). The right-hand side of (14) is in $\mathfrak{B}^{i}$ by the assumption of the induction. Similarly $a(y x)$ is in $\mathfrak{B}^{i}$, and we have $\mathfrak{A}^{i} \mathfrak{B}^{i} \leqq \mathfrak{B}^{i}$. The reciprocal relationship in alternative algebras gives $\mathfrak{B}^{i \mathfrak{A}} \leqq \mathfrak{B}^{i}$, or $\mathfrak{B}^{i}$ is an ideal of $\mathfrak{A}$.

Let $\mathfrak{N}$ be the radical of an alternative algebra $\mathfrak{A}$. Since $\mathfrak{N}$ is nilpotent by Lemma 1, there is a smallest integer $r$ such that $\mathfrak{N}^{r}=0$. Moreover, in the chain

$$
\mathfrak{R}=\mathfrak{N}^{1}>\mathfrak{N}^{2}>\cdots>\mathfrak{N}^{r}=0
$$

the inclusions are proper inclusions, since $\mathfrak{N}^{i-1}=\mathfrak{N}^{i}$ for $2 \leqq i \leqq r$ would imply $\mathfrak{N}^{i-1}=\mathfrak{N}^{i}=\cdots=\mathfrak{N}^{r}=0$, a contradiction. By Lemma 2 the $\mathfrak{N}^{i}$ are ideals of $\mathfrak{A}$.

An inductive argument based on the dimension of $\mathfrak{N}$ suffices to reduce the proof of the principal theorem to the case $\mathfrak{R}^{2}=0$. For the preliminaries disposed of in this section allow us to use the argument of the associative proof $[1, \mathrm{p} .47]$.

3. The case $\mathfrak{N}^{2}=0$. In $\S 4$ we shall prove the principal theorem for $\mathfrak{U}$ having a unity element, with $\mathfrak{R}^{2}=0$ and $\mathfrak{A}-\mathfrak{R}$ a split algebra (that is, either a total matrix algebra or a Cayley-Dickson algebra with 
divisors of zero). At this point let us assume this result and show how the case $\mathfrak{N}^{2}=0$ of the principal theorem may be proved.

First the theorem may be reduced to the case where $\mathfrak{A}$ has a unity element. If $\mathfrak{A}$ has no unity element, adjoin a unity element $\boldsymbol{e}$ to obtain $\mathfrak{A}_{1}=e \mathfrak{F}+\mathfrak{A}$. Then $\mathfrak{A}_{1}$ is alternative. Also the radical $\mathfrak{N}$ of $\mathfrak{A}$ is the radical of $\mathfrak{A}_{1}$. For $\mathfrak{N}$ is a nilpotent ideal of $\mathfrak{A}_{1}$ and is therefore contained in the radical $\mathfrak{N}_{1}$ of $\mathfrak{A}_{1}$. Conversely if $a_{1}$ is in $\mathfrak{N}_{1}$, then $a_{1}=\alpha e+a$ for $\alpha$ in $\mathfrak{F}, a$ in $\mathfrak{A}$, and there exists an integer $t$ such that $a_{1}^{t}=\alpha^{t} e+a^{\prime}=0$ for $a^{\prime}$ in $\mathfrak{A}$. Hence $\alpha^{t}=0, \alpha=0, a_{1}=a$ is in $\mathfrak{A}, \mathfrak{N}_{1} \leqq \mathfrak{A}$. Then $\mathfrak{N}_{1}$ is a nilpotent ideal of $\mathfrak{A}, \mathfrak{R}_{1} \leqq \mathfrak{N}$. If we can obtain a Wedderburn decomposi-

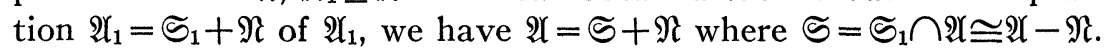

Throughout the remainder of this paper we assume that $\mathfrak{A}$ has a unity element $e$, and that $\mathfrak{N}^{2}=0$. We denote residue classes modulo $\mathfrak{N}$ by the customary bracket symbol [ ].

LEMMA 3. If $\left[u_{1}\right], \cdots,\left[u_{s}\right]$ are pairwise orthogonal idempotents in $\mathfrak{A}-\mathfrak{N}$, there exist pairwise orthogonal idempotents $e_{1}, \cdots, e_{s}$ in $\mathfrak{A}$ such that

$$
\left[e_{i}\right]=\left[u_{i}\right], \quad i=1, \cdots, s .
$$

Furthermore, if $[e]=\left[u_{1}\right]+\cdots+\left[u_{s}\right]$, then $e=e_{1}+\cdots+e_{s}$.

We prove the first assertion by induction. The element

$$
e_{1}=3 u_{1}^{2}-2 u_{1}^{3}
$$

is an idempotent in $\mathfrak{A}$. For, since $u_{1}^{2}-u_{1}$ is in $\mathfrak{N}$, $\mathfrak{N}^{2}=0$, we have $\left(u_{1}^{2}-u_{1}\right)^{2}=0$, and $e_{1}^{2}=e_{1}$ by $(16)$. Also

$$
\left[e_{1}\right]=\left[u_{1}\right]
$$

by (16), so that $e_{1} \neq 0$, and we have proved the case $s=1$. If $f_{1}$ is any idempotent in $\mathfrak{A}$, write $f_{0}=e-f_{1}$, so the Peirce decomposition of $\mathfrak{A}$ relative to $f_{1}$ takes the form

$$
\mathfrak{U}=f_{1} \mathfrak{U} f_{1}+f_{1} \mathfrak{U} f_{0}+f_{0} \mathfrak{U} f_{1}+f_{0} \mathfrak{A} f_{0},
$$

where products $f_{i} a f_{j}(i, j=0,1)$ for $a$ in $\mathfrak{A}$ are trivially seen to be associative. The $f_{i} \mathfrak{A} f_{i}(i=0,1)$ are orthogonal subalgebras of $\mathfrak{A}$. The corresponding Peirce decomposition of $\mathfrak{A}-\mathfrak{N}$ is

$$
\begin{aligned}
\mathfrak{U}-\mathfrak{N}= & {\left[f_{1}\right](\mathfrak{U}-\mathfrak{N})\left[f_{1}\right]+\left[f_{1}\right](\mathfrak{A}-\mathfrak{N})\left[f_{0}\right] } \\
& +\left[f_{0}\right](\mathfrak{U}-\mathfrak{N})\left[f_{1}\right]+\left[f_{0}\right](\mathfrak{U}-\mathfrak{N})\left[f_{0}\right] .
\end{aligned}
$$

We assume the existence of pairwise orthogonal idempotents $e_{2}, \cdots, e_{s}$ in $\mathfrak{A}$ such that $\left[e_{i}\right]=\left[u_{i}\right], i=2, \cdots, s$, and write $f_{1}=e_{2}$ $+\cdots+e_{s}$. Then $\left[u_{1}\right]$ is orthogonal to $\left[f_{1}\right]=\left[u_{2}\right]+\cdots+\left[u_{s}\right]$, and 
we have $\left[u_{1}\right]$ in $\left[f_{0}\right](\mathfrak{A}-\mathfrak{N})\left[f_{0}\right]$. Hence we may choose $u_{1}$ in the subalgebra $f_{0} \mathfrak{H} f_{0}$ of $\mathfrak{A}$, so that $e_{1}$ in (16) is in $f_{0} \mathfrak{U} f_{0}$ and is therefore orthogonal to $f_{1}=e_{2}+\cdots+e_{s}$. That is, $e_{1}, e_{2}, \cdots, e_{s}$ are pairwise orthogonal idempotents in $\mathfrak{A},(15)$ holds, and the inductive proof is complete. Since $e_{1}+\cdots+e_{s}$ is idempotent, $e-\left(e_{1}+\cdots+e_{s}\right)$ is either zero or idempotent. But $[e]=\left[u_{1}\right]+\cdots+\left[u_{s}\right]$ implies that $e-\left(e_{1}+\cdots+e_{s}\right)$ is in $\Re$ and cannot be idempotent; hence $e=e_{1}+\cdots+e_{s}$.

The reduction of the principal theorem to the case $\mathfrak{A}-\mathfrak{N}$ simple is now easy. Let $\mathfrak{A}-\mathfrak{N}=\mathfrak{B}_{1} \oplus \cdots \oplus \mathfrak{B}_{\text {s }}$ for simple algebras $\mathfrak{B}_{i}$. By Lemma 3 , there exist pairwise orthogonal idempotents $\boldsymbol{e}_{\boldsymbol{i}}$ in $\mathfrak{A}$ such that the unity element of $\mathfrak{B}_{i}$ is $\left[e_{i}\right]$. In the refined Peirce decomposition

$$
\mathfrak{A}=\sum \mathfrak{A}_{i j} \quad(i, j=1, \cdots, s)
$$

we have $\mathfrak{A}_{i j}=e_{i} \mathfrak{A} e_{j}$ (associative products!), and the radical $\mathfrak{N}_{i}$ of $\mathfrak{A}_{i i}$ is the intersection of $\mathfrak{N}$ and $\mathfrak{A}_{i i}[9, \S \S 4,5]$. It is easy to see that $\mathfrak{A}_{i i}$ $-\mathfrak{N}_{i} \cong \mathfrak{B}_{i}$. If we have Wedderburn decompositions $\mathfrak{A}_{i i}=\mathfrak{S}_{i}+\mathfrak{N}_{i}$, $\mathfrak{S}_{i} \cong \mathfrak{B}_{i}$, then (since the subalgebras $\mathfrak{A}_{i i}$ of $\mathfrak{A}$ are pairwise orthogonal) the sum of the subalgebras $\mathfrak{S}_{i}$ of $\mathfrak{A}$ is the direct sum $\mathfrak{S}_{=} \mathfrak{S}_{1} \oplus \ldots$ $\oplus \mathfrak{S}_{s} \cong \mathfrak{A}-\mathfrak{N}$, and we have $\mathfrak{A}=\mathfrak{S}+\mathfrak{N}$.

We can now prove the principal theorem. Since $\mathfrak{A}-\mathfrak{N}$ is separable, there exists a splitting field $\Re$ such that $(\mathfrak{A}-\mathfrak{N})_{\Re}=\mathfrak{A}_{\Re}-\mathfrak{N}_{\Re}$ is a direct sum of split algebras. Then $\mathfrak{N}_{\Omega}$ is the radical of $\mathfrak{A}_{\Re}$ (since it is a nilpotent ideal containing the radical). By the result in the preceding paragraph, we may assume that $(\mathfrak{A}-\mathfrak{R})_{\mathfrak{R}}$ has only one simple component (a split algebra). Then by $\S 4$ below, $\mathfrak{A}_{\Re}$ contains a subalgebra $\mathfrak{B} \cong(\mathfrak{A}-\mathfrak{R}) \mathfrak{R}$. The remaining steps are those of the associative proof $[1, \mathrm{p} .48]$, since no form of associativity is used there.

4. The case $\mathfrak{A}-\mathfrak{N}$ a split algebra. We assume throughout that $\mathfrak{A}$ has a unity element $e$, and that $\mathfrak{N}^{2}=0$. Suppose that $\mathfrak{A}-\mathfrak{N}$ is a total matric algebra $\mathfrak{M}_{s}$ of degree $s$. Then the principal theorem is an immediate consequence of Lemma 4, which we state in the following slightly more general form for use in the proof of Lemma 5 .

Lemma 4. Let $\mathfrak{A}-\mathfrak{N}$ contain a total matric algebra $\mathfrak{M}_{s}$ with unity element $[e]$. Then $\mathfrak{A}$ contains a total matric algebra $\mathfrak{M}$ of degree $s$ with unity element $e$, and $\mathfrak{M}_{s}$ is the image of $\mathfrak{M}$ under the homomorphism $x \rightarrow[x]$ of $\mathfrak{A}$ onto $\mathfrak{A}-\mathfrak{N}$.

For let $\mathfrak{M}_{s}$ have basal elements $\left[u_{i j}\right](i, j=1, \cdots, s)$ with the customary multiplication table

$$
\left[u_{i j}\right]\left[u_{k l}\right]=\delta_{j}^{k}\left[u_{i l}\right] \quad \text { (Kronecker delta). }
$$


Then $[e]=\left[u_{11}\right]+\cdots+\left[u_{s s}\right]$, and by Lemma 3 there exist pairwise orthogonal idempotents $e_{i i}$ in $\mathfrak{A}$ such that $\left[u_{i i}\right]=\left[e_{i i}\right], e=e_{11}+\cdots$ $+e_{s s}$. In the Peirce decomposition (18) of $\mathfrak{A}$ relative to this set of idempotents we have $\mathfrak{A}_{i j}=e_{i i} \mathfrak{A} e_{j j}$.

We may take $u_{11}=e_{11}$. Since $\left[u_{i 1}\right]=\left[e_{i i}\right]\left[u_{i 1}\right]\left[e_{11}\right]$, we may also take $u_{i 1}$ in $\mathfrak{A}_{i 1}(i=2, \cdots, s)$, and similarly take $u_{1 j}$ in $\mathfrak{A}_{1 j}(j=2, \cdots, s)$. It follows from (19) that

$$
u_{1 j} u_{j 1}=e_{11}+a_{j}
$$

where $a_{j}$ is in $\mathfrak{N} \cap \mathfrak{A}_{1 j} \mathfrak{A}_{j 1} \leqq \mathfrak{N} \cap \mathfrak{A}_{11}$ since

$$
\mathfrak{A}_{i j} \mathfrak{A}_{j k} \leqq \mathfrak{A}_{i k}
$$

[9, equation (4.5.1)]. Put

$$
e_{1 j}=\left(e_{11}-a_{j}\right) u_{1 j}, \quad e_{i 1}=u_{i 1} \quad(i, j=2, \cdots, s) .
$$

Then $e_{1 j}$ is in $\mathfrak{A}_{11} \mathfrak{A}_{1 j} \leqq \mathfrak{U}_{1 j}$ by $(21)$, and $e_{i 1}$ is in $\mathfrak{A}_{i 1}$. Since

$$
\left(x_{i j} y_{j k}\right) z_{k i}=x_{i j}\left(y_{j k} z_{k i}\right) \quad \text { unless }(i, j, k)=(i, i, i)
$$

for $x_{\alpha \beta}, y_{\alpha \beta}, z_{\alpha \beta}$ in $\mathfrak{A}_{\alpha \beta}[9$, Theorem (4.7.1)], we obtain the formula

$$
e_{1 j} e_{j 1}=e_{11} \quad(j=1, \cdots, s) .
$$

The case $j=1$ of (23) is trivial, and for $j \neq 1$ we have $e_{1 j} e_{j 1}$ $=\left\{\left(e_{11}-a_{j}\right) u_{1 j}\right\} u_{j 1}=\left(e_{11}-a_{j}\right)\left(u_{1 j} u_{j 1}\right)=\left(e_{11}-a_{j}\right)\left(e_{11}+a_{j}\right)=e_{11}$ by $(22)$, (20), and the fact that $e_{11}$ is the unity element of $\mathfrak{A}_{11}$, while $a_{j}^{2}=0$ since $\mathfrak{l}^{2}=0$.

Define

$$
e_{i j}=e_{i 1} e_{1 j} \quad(i \neq j ; i, j=2, \cdots, s)
$$

in $\mathfrak{A}_{i 1} \mathfrak{A}_{1 j} \leqq \mathfrak{A}_{i j}$. Since $\left[e_{i 1}\right]=\left[u_{i 1}\right]$ and $\left[e_{1 j}\right]=\left[e_{11}-a_{j}\right]\left[u_{1 j}\right]=\left[u_{11}\right]\left[u_{1 j}\right]$ $=\left[u_{1 j}\right]$, we have $\left[e_{i j}\right]=\left[u_{i j}\right]$. Note that $(24)$ holds if $i=1$ or $j=1$. Also (24) holds if $i=j$. For $\left(e_{i 1} e_{1 i}\right)^{2}=e_{i 1}\left\{\left(e_{1 i} e_{i 1}\right) e_{1 i}\right\}=e_{i 1} e_{1 i}$ by (23) and the Theorem of Artin; since $\left[e_{i 1} e_{1 i}\right]=\left[u_{i i}\right]=\left[e_{i i}\right]$, we may apply the second part of Lemma 3 to $\mathfrak{A}_{i i}$ to obtain $e_{i i}=e_{i 1} e_{1 i}$. Formula (24) is established for all $i, j=1, \cdots, s$. Now

$$
e_{i j} e_{h k}=0
$$

for $j \neq h$.

For $\mathfrak{A}_{i j} \mathfrak{A}_{h k}=0(j \neq h)$ unless $h=i, k=j[9$, Theorem (4.5.3)]. But

$$
x_{i j}^{2}=0 \quad \text { for } x_{i j} \text { in } \mathfrak{A}_{i j}, i \neq j
$$

$\left[6\right.$, p. 132 , formula $\left.\left(15^{\prime \prime}\right)\right]$, which disposes of the remaining case in (25). We have

$$
e_{1 j} e_{j k}=e_{1 k}, \quad e_{k j} e_{j 1}=e_{k 1} \quad(j, k=1, \cdots, s) .
$$


For, since we have proved (23), we may take $k \neq 1$. Then by (24), (6), (7), (23), and (25), we have $e_{1 j} e_{j k}=e_{1 j}\left(e_{j 1} e_{1 k}\right)=\left(e_{1 j} e_{j 1}\right) e_{1 k}-\left[e_{1 j}\right.$, $\left.e_{j 1}, e_{1 k}\right]=e_{1 k}-\left[e_{1 k}, e_{1 j}, e_{j 1}\right]=e_{1 k}-\left(e_{1 k} e_{1 j}\right) e_{j 1}+e_{1 k}\left(e_{1 j} e_{j 1}\right)=e_{1 k}$. The second equation in (27) is the reciprocal relationship. With (27) we can prove

$$
e_{i j} e_{j k}=e_{i k} \quad(i, j, k=1, \cdots, s) .
$$

For $e_{i j} e_{j k}=\left(e_{i 1} e_{1 j}\right) e_{j k}=e_{i 1}\left(e_{1 j} e_{j k}\right)-\left[\begin{array}{lll}e_{i 1}, & e_{1 j}, & e_{j k}\end{array}\right]=e_{i k}-\left[\begin{array}{lll}e_{1 j}, & e_{j k}, & e_{i 1}\end{array}\right]$ $=e_{i k}-e_{1 k} e_{i 1}+e_{1 j}\left(e_{j k} e_{i 1}\right)$. If $i=k$, this gives $e_{i j} e_{j i}=e_{i i}-e_{11}+e_{1 j} e_{j 1}=e_{i i}$; if $i \neq k$, it gives $e_{i j} e_{j k}=e_{i k}$ by (25). Equations (25) and (28) imply that the subalgebra $\mathfrak{M}$ of $\mathfrak{A}$ with basis $e_{i j}(i, j=1, \cdots, s)$ is a total matric algebra, and the lemma is proved.

Consider the total matric algebra $\mathfrak{M}_{2}$ of degree two over $\mathfrak{F}$; it is a quaternion algebra with divisors of zero. ${ }^{10}$ Taking the usual matric basis $e_{i j}(i, j=1,2)$ for $\mathfrak{M}_{2}$, the unity element is $e_{11}+e_{22}$ and the involution $q \rightarrow \bar{q}=t(q)\left(e_{11}+e_{22}\right)-q$ of $\mathfrak{M}_{2}$ is defined by

$$
\bar{e}_{i i}=e_{j j}, \quad \bar{e}_{i j}=-e_{i j} \quad(i \neq j ; i, j=1,2) .
$$

The algebra $\mathfrak{S}=\mathfrak{M}_{2}+w \mathfrak{M}_{2}$ with elements $q_{1}+w q_{2}$ (for $q_{i}$ in $\mathfrak{M}_{2}$ ) and multiplication

$$
\left(q_{1}+w q_{2}\right)\left(q_{3}+w q_{4}\right)=\left(q_{1} q_{3}+q_{4} \bar{q}_{2}\right)+w\left(\bar{q}_{1} q_{4}+q_{3} q_{2}\right)
$$

is a Cayley-Dickson algebra (we have written $w$ for $v$ in (5) and set $\gamma=1)$. Since the Cayley-Dickson algebra with divisors of zero is unique, it may be taken in this form.

Lemma 5. Let $\mathfrak{A}-\mathfrak{N}=\mathfrak{C}$, a Cayley-Dickson algebra with divisors of zero. Then $\mathfrak{A}=\mathfrak{C}_{1}+\mathfrak{R}$ where $C_{1} \cong C$.

For we may take $\mathfrak{S}$ in the form $\mathfrak{E}=\mathfrak{M}_{2}+[w] \mathfrak{M}_{2}$, where, for elements $[q]$ in $\mathfrak{M}_{2}$, multiplication is given by (30) with brackets. By Lemma 4 , $\mathfrak{A}$ contains a total matric algebra $\mathfrak{M}$ of degree two such that $e$ is the unity element of $\mathfrak{M}$ and the matric basis $e_{i j}$ of $\mathfrak{M}$ gives the matric basis $\left[e_{i j}\right]$ of $\mathfrak{M}_{2}$. Note that $\overline{[q]}=[\bar{q}]$ for $q$ in $\mathfrak{M}$. In the proof of $[\mathbf{5}$, Theorem 2], it is shown that the multiplication formula (5) follows from the alternative law and formulas $v^{2}=\gamma e, q v=v \bar{q}$ for $q$ in $\mathfrak{Q}$. Therefore, in order to prove the lemma, it is sufficient to show the existence of an element $v$ not in $\mathfrak{M}$ such that

$$
v^{2}=e, \quad q v=v \bar{q}
$$

for $q$ in $\mathfrak{M}$.

Write

$$
\left[f_{i j}\right]=[w]\left[e_{j i}\right] \quad \text { for } i \neq j(i, j=1,2) .
$$

\footnotetext{
${ }^{10}$ See footnote 4.
} 
We may take $f_{i j}$ in $\mathfrak{A}_{i j}(i \neq j)$, for $\left[e_{i i}\right]\left(\left[f_{i j}\right]\left[e_{j j}\right]\right)=\left[e_{i i}\right]\left([w]\left[e_{j j}\right]^{2}\right)$ $=[w]\left[\bar{e}_{i i} e_{j j}\right]=[w]\left[e_{j j}\right]=\left[f_{i j}\right]$ by (32), (30), (29). Now $\left[e_{j i}\right]\left[f_{i j}\right]$ $=\left[e_{j i}\right]\left([w]\left[e_{j j}\right]\right)=-[w]\left(\left[e_{j i}\right]\left[e_{j j}\right]\right)=[0]$ implies that

$$
e_{j i} f_{i j}=c_{j}
$$

for $c_{j}$ in $\mathfrak{R} \cap \mathfrak{A}_{j j}$. Write

$$
h_{i j}=f_{i j}-e_{i j} c_{j} .
$$

Then $h_{i j}$ is in $\mathfrak{A}_{i j}$, $\left[h_{i j}\right]=\left[f_{i j}\right]$, and

$$
e_{j i} h_{i j}=h_{i j} e_{j i}=0 \quad(i \neq j ; i, j=1,2) .
$$

For $e_{j i} h_{i j}=c_{j}-e_{j i}\left(e_{i j} c_{j}\right)=c_{j}-\left(e_{j i} e_{i j}\right) c_{j}=0$ by (22). Moreover, $e_{i j} c_{j}$ $=e_{i j}\left(e_{j i} f_{i j}\right)=\left(e_{i j} e_{j i}\right) f_{i j}-\left[e_{i j}, e_{j i}, f_{i j}\right]=f_{i j}+\left[f_{i j}, e_{j i}, e_{i j}\right]=f_{i j}+\left(f_{i j} e_{j i}\right) e_{i j}$ $-f_{i j}\left(e_{j i} e_{i j}\right)=\left(f_{i j} e_{j i}\right) e_{i j}$ by $(7)$, and we have $h_{i j} e_{j i}=f_{i j} e_{j i}-\left\{\left(f_{i j} e_{j i}\right) e_{i j}\right\} e_{j i}$ $=0$ by (22). Now $\left[h_{i j}\right]\left[h_{j i}\right]=\left[f_{i j}\right]\left[f_{j i}\right]=\left[e_{i i}\right]\left[\bar{e}_{j j}\right]=\left[e_{i i}\right]$ implies that

$$
h_{i j} h_{j i}=e_{i i}+a_{i}
$$

for $a_{i}$ in $\mathfrak{R} \cap \mathfrak{A}_{i i}(i=1,2)$. Since $\mathfrak{N}^{2}=0$, we have $a_{\mathfrak{i}}^{2}=0$, and

$$
\left(e_{i i}-a_{i}\right)\left(e_{i i}+a_{i}\right)=e_{i i}=\left(e_{i i}+a_{i}\right)\left(e_{i i}-a_{i}\right) .
$$

Write

$$
p_{12}=\left(e_{11}-a_{1}\right) h_{12}, \quad p_{21}=h_{21} .
$$

Then $p_{i j}$ is an $\mathfrak{A}_{i j},\left[p_{i j}\right]=\left[f_{i j}\right]$, and we shall prove

$$
p_{i j} p_{i i}=e_{i i} \quad(i \neq j ; i, j=1,2) .
$$

For $p_{12} p_{21}=\left\{\left(e_{11}-a_{1}\right) h_{12}\right\} h_{21}=\left(e_{11}-a_{1}\right)\left(h_{12} h_{21}\right)=\left(e_{11}-a_{1}\right)\left(e_{11}+a_{1}\right)=e_{11}$ by (22) and (34). But $a_{i} h_{i j}=\left(h_{i j} h_{j i}-e_{i i}\right) h_{i j}=h_{i j}\left(h_{j i} h_{i j}\right)-h_{i j}$ $=h_{i j}\left(e_{j j}+a_{j}\right)-h_{i j}=h_{i j} a_{j}$, so that

$$
p_{12}=h_{12}-a_{1} h_{12}=h_{12}-h_{12} a_{2}=h_{12}\left(e_{22}-a_{2}\right)
$$

and $p_{21} p_{12}=h_{21}\left\{h_{12}\left(e_{22}-a_{2}\right)\right\}=\left(h_{21} h_{12}\right)\left(e_{22}-a_{2}\right)=\left(e_{22}+a_{2}\right)\left(e_{22}-a_{2}\right)=e_{22}$ by (22) and (34). It follows from (33), (36), and (22) that

$$
e_{i j} p_{j i}=p_{j i} e_{i j}=0 \quad(i \neq j ; i, j=1,2) .
$$

Finally write

$$
v=p_{12}+p_{21}
$$

Then $[v]=\left[f_{12}\right]+\left[f_{21}\right]=[w]$, and $v^{2}=\left(p_{12}+p_{21}\right)^{2}=p_{12} p_{21}+p_{12} p_{21}=e_{11}$ $+e_{22}=e$ by (26) and (35). For $i \neq j$ we have $e_{i i} v=e_{i i}\left(p_{i j}+p_{j i}\right)=p_{i j}$ $=\left(p_{i j}+p_{j i}\right) e_{j j}=v e_{j j} ;$ also (26) implies that $\left(e_{i j}+p_{i j}\right)^{2}=e_{i j} p_{i j}+p_{i j} e_{i j}=0$ so that $e_{i j} v=e_{i j}\left(p_{i j}+p_{j i}\right)=e_{i j} p_{i j}=-p_{i j} e_{i j}=-\left(p_{i j}+p_{j i}\right) e_{i j}=-v e_{i j}$ by 
(37). Hence $q v=v \bar{q}$ for all $q$ in $\mathfrak{M}$ by (29), and we have shown the existence of an element $v$ in $\mathfrak{A}$, not in $\mathfrak{M}$, satisfying (31). This completes the proof of the lemma, and of the theorem.

\section{REFERENCES}

1. A. A. Albert, Structure of algebras, Amer. Math. Soc. Colloquium Publications, vol. $24,1939$.

2. - Non-associative algebras I. Fundamental concepts and isotopy, Ann. of Math. vol. 43 (1942) pp. 685-707.

3. - Non-associative algebras II. New simple algebras, Ann. of Math. vol. 43 (1942) pp. 708-723.

4. I. M. H. Etherington, Special train algebras, Quart. J. Math. Oxford Ser. vol. 12 (1941) pp. 1-8.

5. R. D. Schafer, Alternative algebras over an arbitrary field, Bull. Amer. Math. Soc. vol. 49 (1943) pp. 549-555.

6. M. Zorn, Theorie der alternativen Ringe, Abh. Math. Sem. Hamburgischen Univ. vol. 8 (1930) pp. 123-147.

7. - Alternativkörper und quadratische Systeme, Abh. Math. Sem. Hamburgischen Univ. vol. 9 (1933) pp. 395-402.

8. - On a theorem of Engel, Bull. Amer. Math. Soc. vol. 43 (1937) pp. 401404.

9. - Alternative rings and related questions I: Existence of the radical, Ann. of Math. vol. 42 (1941) pp. 676-686.

The Institute for Advanced Study 